\title{
Priority-Based Human Resource Allocation in Business Processes ${ }^{\star}$
}

\author{
Cristina Cabanillas ${ }^{1}$, José María García ${ }^{2}$, Manuel Resinas ${ }^{3}$, \\ David Ruiz ${ }^{3}$, Jan Mendling ${ }^{1}$, and Antonio Ruiz-Cortés ${ }^{3}$ \\ 1 Vienna University of Economics and Business, Austria \\ \{cristina.cabanillas, jan.mendling\}@wu.ac .at \\ 2 STI Innsbruck, University of Innsbruck, Austria \\ jose.garcia@sti2.at \\ 3 University of Seville, Spain \\ \{resinas, druiz, aruiz\}@us.es
}

\begin{abstract}
In Business Process Management Systems, human resource management typically covers two steps: resource assignment at design time and resource allocation at run time. Although concepts like rolebased assignment often yield several potential performers for an activity, there is a lack of mechanisms for prioritizing them, e.g., according to their skills or current workload. In this paper, we address this research gap. More specifically, we introduce an approach to define resource preferences grounded on a validated, generic user preference model initially developed for semantic web services. Furthermore, we show an implementation of the approach demonstrating its feasibility.
\end{abstract}

Keywords: preference modeling, preference resolution, priority-based allocation, priority ranking, RAL, resource allocation, SOUP.

\section{Introduction}

Business Process Management System (BPMS) are increasingly used for supporting service composition. Typically, they work with executable process models that define the control flow, data processing, and resource involvement of a specific process. Resources in this context include both automatic services and services provided by human resources. In particular, the appropriate selection of human resources is critical as various factors such as workload or skills have an impact on work performance. While priorities for automatic services are intensively researched, it is surprising that prioritizing human resources has been hardly discussed. In classical workflow management, only two steps of resource management are considered: resource assignment at the level of process specification and resource allocation at run time [1]. Resource assignment builds on

\footnotetext{
* This work was partially supported by the European Union's Seventh Framework Programme (FP7/2007-2013), the European Commission (FEDER), the Spanish and the Andalusian R\&D\&I programmes (grants 318275 (GET Service), 284860 (MSEE), TIN2009-07366 (SETI), TIN2012-32273 (TAPAS), TIC-5906 (THEOS)).
} 
defining for each activity the human resources that are candidates to work on the activity. These are called potential performers. At run time, the resource allocation step considers these potential performers to select actual performers (often a single person) who find the activity in their worklist. For instance, the Yet Another Workflow Language (YAWL) system [2] uses this concept to support various Workflow Resource Patterns (WRPs) 3]. Even though theseWRPs identify strategies to balance workload, there is no explicit consideration of prioritizing potential performers to facilitate the selection of the actual performer. This is remarkable, as deriving a set of performers immediately poses the question of who would be the best candidate to pick up the work. Also professional solutions such as Activit:11, WebSphere MQ2 or BPEL4People, do not provide means to prioritize performers, but assign priority indicators to activities only.

In this paper, we address this research gap of how prioritization of resources can be integrated in BPMS. More specifically, we provide two contributions: (i) we conceptually define prioritized allocation based on preferences; and (ii) we propose a concrete way in which preferences over resources can be defined such that a resource priority ranking can be automatically generated. Our solution builds on the adaptation of a user preference model that was developed for the discovery and ranking of semantic web services [4] to the domain at hand. As a proof of concept, we have extended the resource management tool Collection of Resource-centrIc Supporting Tools And Languages (CRISTAL) with the Semantic Ontology of User Preferences (SOUP) component [6] to support priority-based allocation. In this system, Resource Assignment Language (RAL) [7] is used for resource selection.

The rest of the paper is structured as follows. In Section 2 , we conceptually define priority-based allocation based on preferences and describe the requirements to address it based on a motivating scenario. In Section 3, we explain the adaptation of the preference metamodel and its formalization for automated prioritization. In Section 4, we evaluate the approach regarding preference modeling and resolution. Work related to preference modeling and resource prioritization in Business Processes (BPs) is detailed in Section [5] before closing the paper by drawing some conclusions and deriving potential future work in Section 6 .

\section{Priority-Based Resource Allocation}

We define priority-based resource allocation as the ability to rank a set of resources according to one or more preferences. The result is thus a prioritized list of resources that can be allocated to a process activity. In the following, we present a real scenario that motivates the problem, and a set of requirements that must be considered in order to deal with it.

\footnotetext{
${ }^{1}$ http://www.activiti.org/

2 http://www-01.ibm.com/software/integration/wmq/

3 http://www.isa.us.es/cristal
} 


\subsection{Motivating Scenario}

The need for resource prioritization is motivated by a real scenario located in the Andalusian Institute of Public Administration (in Spanish IAAP), which serves more than eight million end users. The Business Process (BP) in question represents the procedure to create and process a resolution proposal for hiring people and has a high use frequency in the IAAP. Fig. 11 shows the process in Business Process Model and Notation (BPMN), Once a draft of a resolution proposal is created, it is concurrently sent to the Consultative Board and to the Legal Department for evaluation. The IAAP then analyzes the reports and decides whether an external resolution is required. In that case, a request is sent to an external committee, which must create and send a new resolution. Otherwise, the resolution proposal is reviewed, and changes are applied to the initial one according to the reports received. In any case, the documents generated are signed and archived, and the resolution result is appropriately notified.

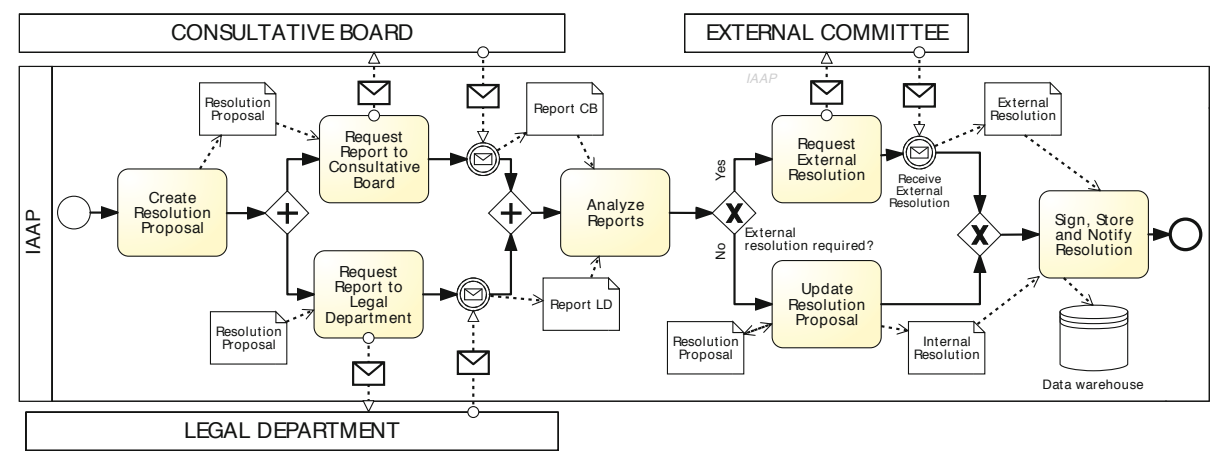

Fig. 1. BP to create and process a resolution proposal for hiring people

The part of the organizational structure of the IAAP related to Administrative Resource Management involved in this $\mathrm{BP}$ is hierarchically structured in eight positions: Business Manager (BM), Technician of the IAAP (T-IAAP), Assistant of the IAAP (A-IAAP), Secretary (SE), Assistant of the Legal Department (ALD), Technician of the Legal Department (T-LD), Assistant of the Consultative Board (A-CB) and Technician of the Consultative Board (T-CB). They are occupied by a total of eleven people. Table 1 shows the selection conditions for each activity (positions are acronymized) and the preferences to prioritize the resulting set of potential performers for allocation.

\subsection{Requirements for Resource Prioritization}

The preferences for priority-based allocation as defined in the example have to yield a partial order relation over the set of potential performers of an activity. 
Table 1. Resource selection conditions and resource preferences

\begin{tabular}{|l|l|l|}
\hline Activity & $\begin{array}{l}\text { Potential } \\
\text { Performers }\end{array}$ & Preferences \\
\hline $\begin{array}{l}\text { Create Resolu- } \\
\text { tion Proposal }\end{array}$ & T-IAAP & $\begin{array}{l}\text { The greatest number of past executions of the ac- } \\
\text { tivity (history) }\end{array}$ \\
\hline $\begin{array}{l}\text { Request Report } \\
\text { to CB/LD }\end{array}$ & T-IAAP & $\begin{array}{l}\text { The best balance between low cost } \text { price) and short } \\
\text { worklist ( availability), i.e., a person is preferred over } \\
\text { another person if his/her cost is lower and his/her } \\
\text { worklist is not longer, or vice versa }\end{array}$ \\
\hline Analyze Reports & T-IAAP & $\begin{array}{l}\text { The least average execution time for the activity } \\
\text { (speed) }\end{array}$ \\
\hline $\begin{array}{l}\text { Request External } \\
\text { Resolution }\end{array}$ & SE & $\begin{array}{l}\text { The best skills on using a specific software applica- } \\
\text { tion (expertise) }\end{array}$ \\
\hline $\begin{array}{l}\text { Update Resolu- } \\
\text { tion Proposal }\end{array}$ & $\begin{array}{l}\text { T-IAAP, T- } \\
\text { CB or T-LD }\end{array}$ & $\begin{array}{l}\text { The same person who created the resolution draft } \\
\text { Binding of Duties (BoD) [8] } / \text { ) }\end{array}$ \\
\hline $\begin{array}{l}\text { Sign, Store and } \\
\text { Notify Resolu- } \\
\text { tion }\end{array}$ & SE & $\begin{array}{l}\text { A person that has been working for the company for } \\
\text { at least one year (experience). In case of no distinc- } \\
\text { tion, the person with shortest worklist (availability) }\end{array}$ \\
\hline
\end{tabular}

More specifically, we identify a set of requirements that must hold in order to deal with such automated resource prioritization as follows:

- Resource assignment. Some mechanism for resource assignment must be put into place to calculate the potential performers before resource prioritization. There are many different approaches for that purpose, e.g., [8 10].

- Expressive preference modeling. In this context, there is a need for expressive preferences that range from single-value criteria (e.g., age), to composite preferences where the preferences themselves can be ranked (cf. preference for Sign, Store and Notify Resolution in Table 1).

- Preference resolution. Some mechanism must enable the automated resolution of preferences at run time based on actual values.

- Information availability. In addition, similarly as for resource assignment, specific information (also called properties) about the resources needs to be stored, updated, and retrieved, to define and resolve resource preferences. For instance, the following types of properties can be distinguished for our motivating scenario:

- Personal and Organizational Data. From personal data such as the ID number, name or age, to properties related to positions occupied in the company or functional roles being held. This data partially depends on the type of organizational model used in the company.

- Skills. As shown for activity Request External Resolution in Table 1. knowledge on specific software applications, technologies, methodologies, etcetera may be of interest for resource prioritization.

- Professional Information. Information on the salary of the resource (e.g. in terms of cost/hour) or the years of experience, can be necessary to determine the resource that best meets the needs of the company. 
- Worklist. The workload of the resources can also be critical to offer or allocate an activity to a specific person. An organization may prefer having balanced workloads rather than very busy people and idle people.

- History. For each person, information about the past execution of process activities must be stored, e.g. activities performed, BPs to which they belonged, execution time, number of times executed, etcetera.

This list cannot be exhaustive, but has to be adapted for the context of the process at hand. For instance, in other cases it might also be necessary to access calendar data, such as scheduled meetings or holidays, in order to prioritize according to availability on a specific date.

\section{Materializing Priority-Based Resource Allocation}

As derived from the previous section, the main challenge for resource prioritization is two-fold: (i) to come up with a mechanism to express preferences over resources, and (ii) to develop a way to rank a set of resources according to those preferences. Furthermore, the solution should be as independent as possible from the properties used in the preferences so that they can accommodate the different requirements several organizations may have.

The first challenge is solved by leveraging SOUP, a highly expressive user preference model defined by García et al. [4, 6] that we adapt to the BP domain in this paper. To deal with the second challenge, we have developed a novel algorithm to rank resources according to the preferences expressed in SOUP. Both the preference metamodel and the algorithm are independent of the properties used in the preferences.

\subsection{SOUP: A Metamodel to Define Preferences}

In SOUP, a preference can be intuitively expressed as "I prefer $y$ rather than $x$ ", where $x$ and $y$ are instances of domain concepts that represent properties of the resources to be allocated (e.g., size of the worklist). This relation between concept instances can be mathematically interpreted as a strict partial order. Therefore, in SOUP a preference can be defined as:

Definition 1 (Preference). Let $\mathcal{C}$ be a non-empty set of domain concepts, and $\operatorname{dom}(\mathcal{C})$ the set of all possible instances of those concepts. We define a preference as $\mathcal{P}=\left(\mathcal{C},<^{\mathcal{P}}\right)$, where $<^{\mathcal{P}} \subseteq \operatorname{dom}(\mathcal{C}) \times \operatorname{dom}(\mathcal{C})$ is a strict partial order (irreflexive, transitive and asymmetric), and if $x, y \in \operatorname{dom}(\mathcal{C})$, then $x<^{\mathcal{P}} y$ is interpreted as "I prefer y rather than $\mathrm{x}$ ".

Consequently, each preference term instance defines its order depending on the concrete concepts referred $(\mathcal{C})$ and some operand values that determine the evaluation of the $<^{\mathcal{P}}$ relation. Furthermore, if we consider a finite set of concept instance pairs $(x, y) \in<^{\mathcal{P}}, \mathcal{P}$ can be represented as a directed acyclic graph, also known as Hasse diagram [11], where each node corresponds to a concept instance, and edges represent the preference relation $<^{\mathcal{P}}$. 
Fig. 2 shows a UML representation of SOUP preference terms. Atomic preferences can be expressed using different preference terms, whereas composite preferences can be used to compose those terms, defining the relation between previously expressed atomic preferences. Both atomic and composite preferences are handled by ranking mechanisms that implement the ranking process according to the corresponding term definition.

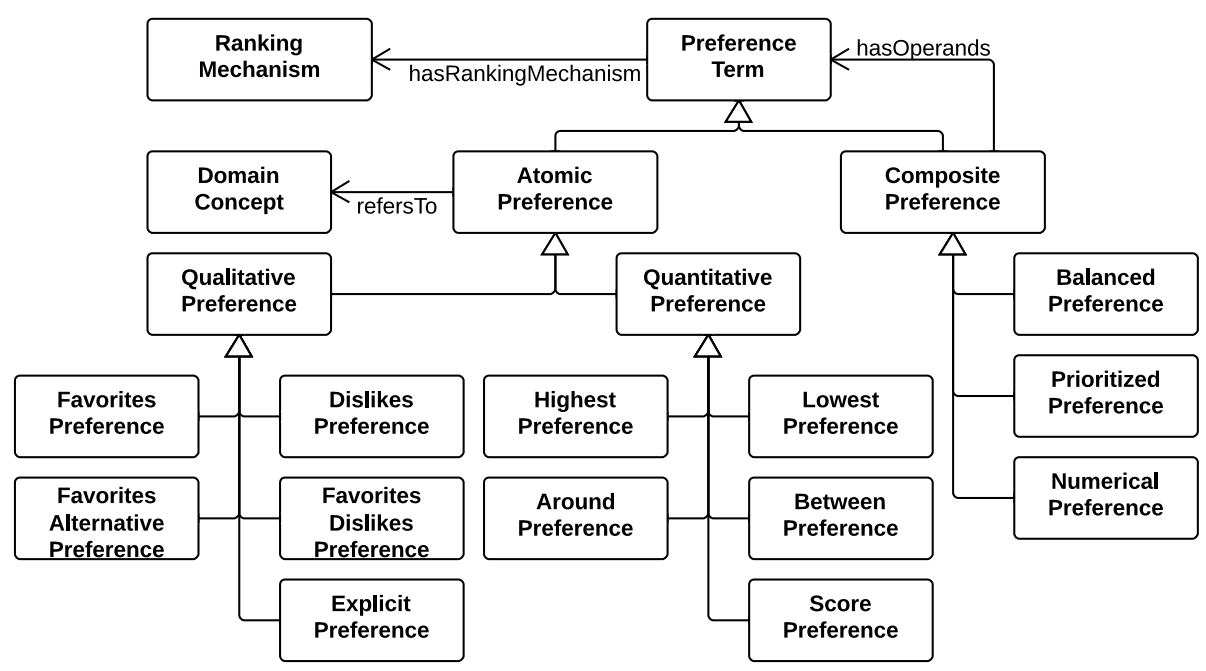

Fig. 2. UML representation of SOUP

In particular, atomic preferences are related to a domain-specific concept that usually represents a property that should be optimized to fulfill the user preference over it. SOUP supports both qualitative and quantitative preferences, depending on the nature of the property referred by the concrete preference. On the one hand, if the property is qualitative, e.g. the skills of a resource, one can use a Favorites preference to state that certain values of that property are favored against the rest (e.g., skills on a concrete software application). Conversely, a Dislikes preference can be used to enumerate the values that should not be provided for the referred property. A FavoritesAlternative allows defining a favorite and an alternative set of property values, meaning that values contained in the former set are the most preferred, but if there is none then values from the latter set can also be considered. A FavoritesDislikes preference is a combination of a Favorites and a Dislikes preference, where preferred values are the ones in the favored set or at least not in the disfavored set. Finally, an Explicit preference simply states the preference between two concrete values of a property (e.g. skills on LibreOffice are more preferred than skills on Microsoft Office).

On the other hand, quantitative preferences compare numerical values of the related properties. Thus, a Highest (Lowest) preference means the user prefers higher (lower) values for the referred property. Around and Between preferences 


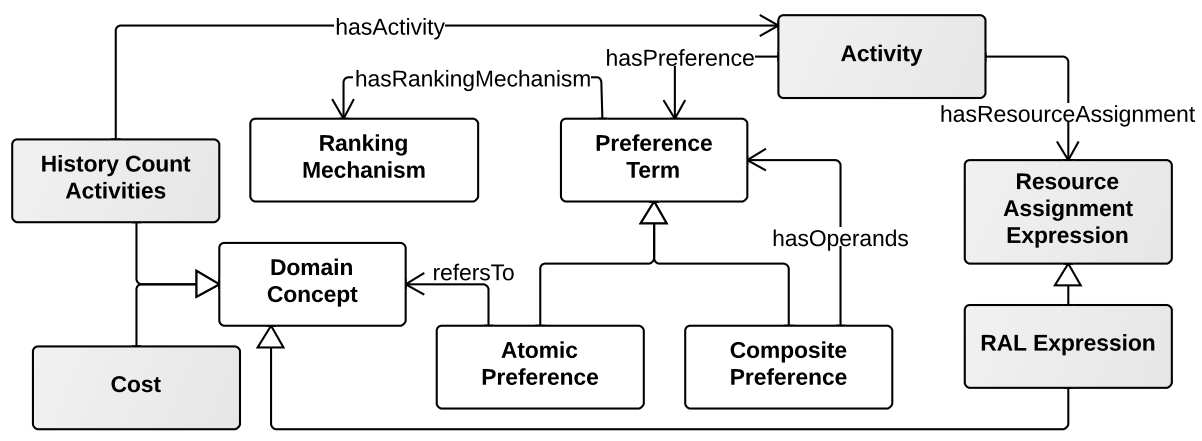

Fig. 3. Modeling priority-based resource allocation using SOUP

prefer values that are close to a specific value, or included within an interval, respectively. Finally, a Score preference expresses that the property value will be evaluated using a scoring function that returns a real value between 0 and 1 expressing to what extent the referred property value is preferred against others.

Concerning composite preferences, SOUP provides three facilities for defining the semantics. First, a Balanced preference $\mathcal{P}$ combines preference terms $\mathcal{P}_{1}, \ldots, \mathcal{P}_{n}$ using the Pareto-optimality principle. Therefore, a resource $r s c_{l}$ is considered better than another resource $r s c_{m}$ with respect to $\mathcal{P}$ if $r s c_{l}$ is better than $r s c_{m}$ with respect to any $P_{i}$ such that $r s c_{l}$ is not worse than $r s c_{m}$ with respect to the rest of the combined preferences $\mathcal{P}_{j}$ with $i \neq j$.

Second, a Prioritized preference combines preferences in importance order. If a list of terms $\mathcal{P}_{1}, \mathcal{P}_{2}, \ldots, \mathcal{P}_{n}$ is combined using this operator, resources will be ranked first in terms of $\mathcal{P}_{1}$. Those that are equally preferred using $\mathcal{P}_{1}$ are ranked in terms of $\mathcal{P}_{2}$, and so forth.

Finally, a Numerical preference is the combination of preferences using a real function to obtain a numerical score value for each resource. Resources are ranked in terms of their score values. However, this composite preference can only combine quantitative preferences that can be evaluated to a score value.

\subsection{Modeling Priority-Based Resource Allocation with SOUP}

Two aspects must be modeled for resource prioritization, namely resource assignments for defining potential performers, and preference modeling for defining their order of priority (cf. Fig. 3).

First, each activity of the $\mathrm{BP}$ has a resource assignment expression that defines its set of potential performers. In our case, we use Resource Assignment Language (RAL) [12] as a language to select a set of potential performers. The language is grounded on a consensual organizational metamodel [13] that takes into consideration not only people and roles, but also positions, organizational units and skills. It also allows selecting people based on the performers of previous activities in the process. We have chosen Resource Assignment Language (RAL) for its expressiveness, extensibility, and for its capabilities to automatically resolve RAL expressions [7]. 
Second, preferences for resource prioritization are formulated using SOUP Preference Terms. All such preferences in SOUP must refer to some domain concept that represents the properties used in the preference. Therefore, modeling the preferences specified for each of the activities of the scenario also involves identifying the domain concepts used to prioritize resources. For instance, our scenario requires domain concepts like Cost and the number of times a resource has carried out a certain activity (History Count Activities). Additionally, a RAL expression can also be used in place of a domain concept. An example of such a preference is I prefer the resource that is responsible for activity Create Resolution Proposal, which can be modeled by means of a preference term of type Favorites referred to a domain concept of type RAL Expression and an operand that specifies the expression: IS PERSON RESPONSIBLE FOR ACTIVITY Create Resolution Proposal.

\subsection{Ranking Resources According to SOUP Preferences}

The prioritization of resources according to preferences is based on ranking mechanisms, which can be defined as follows:

Definition 2 (Ranking mechanism). We define a ranking mechanism as an algorithm that receives as input a preference and a set of resources to be ranked, and returns as output a partially ordered set of the resources ranked according to the preference.

Each Preference Term has a ranking mechanism that is used to implement the ranking process according to the corresponding term definition. Ranking mechanisms can be shared between different preference terms. In [ []], ranking mechanisms for composite preference terms are discussed. However, the authors do not provide details about the ranking mechanisms for atomic preferences because they heavily depend on the characteristics of the knowledge base that contains the information about the domain concepts used in the preferences.

Therefore, in this section we adapt SOUP by providing a formalization of a generic ranking mechanism for atomic preferences, which has been designed to deal with the following characteristics of the knowledge base used for prioritybased resource allocation: (1) it is usually distributed in different heterogeneous repositories such as a process $\log$, an organizational model and an Enterprise Resource Planning (ERP) system; (2) it is dynamic in the sense that some of its repositories are continously changing (e.g., the worklist of each resource); and (3) computing the value of a domain concept for a resource can be a complex operation. For instance, computing the availability of a resource may involve checking his/her agenda, a calendar of the holidays of the country in which s/he works and the worklist of his/her pending tasks.

Reflecting these characteristics, we separate the evaluation of a domain concept for a resource from the ordering of the resources according to this evaluation. The first task is solved by defining a function vval $_{\mathcal{P}}^{K B}$ that is specific for each domain concept and for each knowledge base and can be defined as follows. 
Definition 3 (Domain concept evaluation). Let $K B$ be a knowledge base, $\mathcal{P}=\left(\mathcal{C},<^{\mathcal{P}}\right)$ be an atomic preference, and $\mathcal{R}$ be the set of resources of the organization.

- If the domain concept $\mathcal{C}$ represents a quantitative property, then we define the evaluation as a function eval ${ }_{\mathcal{P}}^{K B}: \mathcal{R} \rightarrow \mathbb{R}$, such that it returns a real number that represents the value of the domain concept for the given resource.

- If the domain concept $\mathcal{C}$ represents a qualitative property, then we define the evaluation as a function eval $\mathcal{P}^{K B}: \mathcal{R} \rightarrow\{$ false, true $\}$, such that it returns a boolean that represents whether the value of the domain concept belongs to the set specified in the preference.

For instance, the evaluation of the quantitative concept size of the worklist for a resource $r$ is the size of the worklist of resource $r$. Similarly, the evaluation of the qualitative concept resource expression with operand IS PERSON RESPONSIBLE FOR ACTIVITY Create Resolution Proposal (which is a RAL expression) for a resource $r$ is true if, according to the organizational model, $r$ is a person responsible for that activity.

The second task that must be performed involves applying the partial order specified by the type of a preference to the result of an evaluation. This is done by defining for each type of preference, a function that compares these results.

Definition 4 (Greater-than comparison). Let $K B$ be a knowledge base with information about the domain concepts used in the preferences, $\mathcal{P}$ be an atomic preference such that $\mathcal{P}=\left(\mathcal{C},<^{\mathcal{P}}\right)$, and $D$ be the range of function eval ${ }_{\mathcal{P}}^{K B}$ for such preference. We define a greater-than comparison for partial order $<^{\mathcal{P}}$ as a function $g t_{<_{\mathcal{P}}}: D \times D \rightarrow\{$ false, true $\}$ such that $g t_{<_{\mathcal{P}}}\left(d_{i}, d_{j}\right)$ returns true if $d_{i}$ is greater than $d_{j}$ according to $<^{\mathcal{P}}$, i.e., if $d_{i}$ is preferred over $d_{j}$.

The implementation of this function can be straightforwardly derived from the type of the preference it corresponds to. For instance, if $<^{\mathcal{P}}$ corresponds to the type Higher, then $g t_{\text {Higher }}\left(n, n^{\prime}\right)$ is true iff $n>n^{\prime}$. The same procedure can be applied to all of the other types of atomic preferences.

Using these two functions it is easy to provide an implementation of a generic ranking mechanism of atomic preferences as depicted in Algorithm 1. The algorithm simply iterates over the list of resources provided to the ranking mechanism and adds an edge $r \rightarrow r^{\prime}$ to the graph if $r^{\prime}$ is preferred to $r$ according to the preference $\mathcal{P}$ and the information in the knowledge base $K B$. Note that it is possible to provide more efficient implementations by means of domain-specific ranking mechanisms that leverage capabilities of the repositories of the knowledge base. However, a discussion of the different implementations of ranking mechanisms that can be developed is out of the scope of this paper.

Regarding ranking mechanisms for composite preferences, a similar approach could be followed. For instance, a ranking mechanism for composite preference Prioritized could be implemented using the same algorithm as Algorithm 1 but changing function $g t_{<\mathcal{P}}$ for a function that uses the ranking mechanisms of its composed preferences to obtain partial results and, then, composes all of them together according to the semantics of Prioritized. 


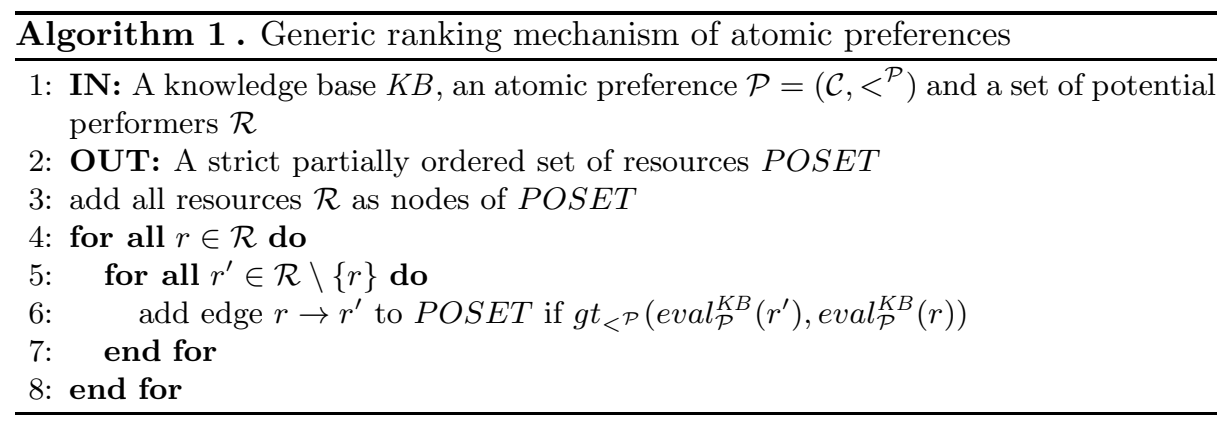

Finally, having all of these ranking mechanisms, the priority-based allocation for an activity $A$ of a $\mathrm{BP}$ just involves using the appropriate ranking mechanism with the allocation preferences of such an activity and the set of resources selected during the resource assignment as inputs. Then, from the partially ordered set obtained by the ranking mechanism it is easy to derive a total order that is a linear extension of the partial order using well-known topological sorting algorithms [14]. Note that preferences define a strict partial order amongst the resources. This means that given two resources $r_{1}$ and $r_{2}$ it may not be possible to establish a preference between them. In that case, the resources are randomly ordered.

\section{Evaluation}

To validate our proposal, we have developed a proof-of-concept implementation 4 for priority-based allocation and we have applied it to the scenario detailed in Section 2.1. The implementation can be divided into a domain-independent part and a domain-specific part. The first part is based on PURI [6], a preference framework that provides the building blocks to implement new ranking mechanisms and also provides an implementation of the ranking mechanisms of all of the composite preferences. For our proof-of-concept, we implemented the generic ranking mechanism for both the atomic preferences and for all $g t$ functions based on the preference types defined in SOUP. The second part involves two steps: (1) identifying the domain concepts used to prioritize resources and modeling the preferences for each activity of the process, and (2) identifying the knowledge base and implementing the eval functions to evaluate the domain concepts. We discuss these two steps in the following.

\subsection{Modeling the Preferences}

Modeling the preferences specified for each of the activities of the scenario involves identifying the domain concepts used to prioritize resources, and using the SOUP metamodel to express the preferences regarding such domain concepts.

\footnotetext{
${ }^{4}$ Available at http://www.isa.us.es/cristal
} 


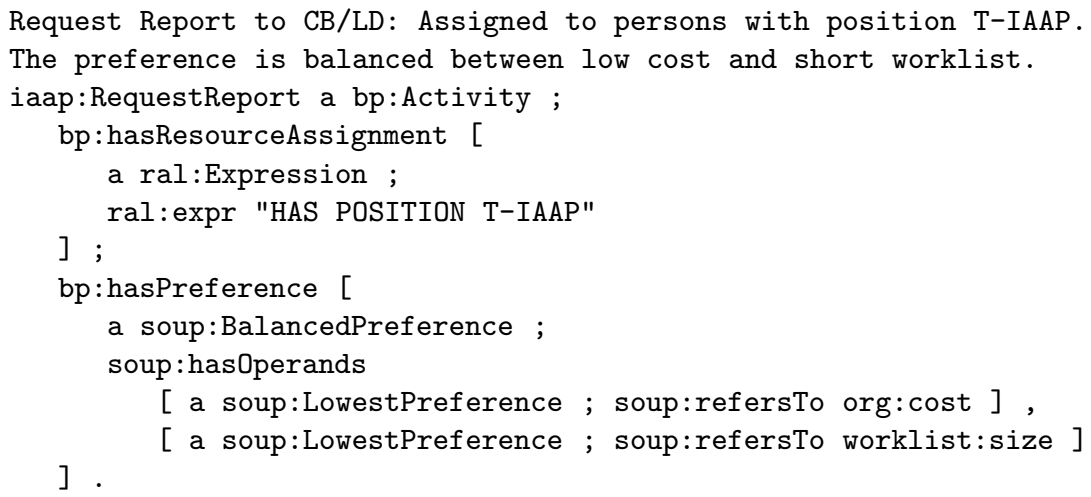

Update Resolution Proposal: Assigned to people with positions T-IAAP, T-CB or T-LD. The preferred person is that who did the initial proposal. iaap:UpdateProposal a bp:Activity ;

bp:hasResourceAssignment [

a ral:Expression ;

ral:expr "HAS POSITION T-IAAP OR HAS POSITION T-CB OR

HAS POSITION T-LD"

] ;

bp:hasPreference [

a soup:FavoritesPreference ;

soup:refersTo ral:Expression ;

soup:hasFavorites "IS PERSON RESPONSIBLE FOR ACTIVITY

Create Resolution Proposal Draft"

].

Fig. 4. Examples of preferences expressed in RDF/Turtle syntax

Due to space constraints, we illustrate how these two tasks have been done with regard to activities Request Report to $C B / L D$ and Update Resolution Proposal. The same approach can be used with the remaining activities.

Fig. 4 depicts the resource assignment (bp: hasResourceAssignment) and the preferences (bp: hasPreferences) for the aforementioned activities expressed in SOUP using RDF/Turtle syntax. For activity Request Report to $C B / L D$ the preference is composite because it balances two atomic preferences: lowest cost and shortest worklist. Therefore, the composite preference is represented by means of an element of type soup:BalancedPreference, which is the type of composite preference that best suits the intention of the modeler. Regarding the atomic preferences, they are connected with the composite preference by means of relation soup:hasOperands. Both atomic preferences are of the same type (soup:LowestPreference). However, the former refers to the domain concept expressed with org:cost, which represents the cost of the resource, whereas the latter refers to the domain concept expressed with worklist:size, which represents the size of the worklist of the resource. Note that these two domain 
concepts must have an eval function implemented for them so that they can be evaluated for each resource.

The preference for activity Update Resolution Proposal is also atomic. However, it refers to the organizational information stored about each resource. In particular, it sets a qualitative preference stating that it prefers the people that fulfill the condition stated by property soup:hasFavorites.

\subsection{Identifying the $\mathrm{KB}$ and Implementing eval Functions}

In our scenario, there are three repositories that store the values of the properties used for the prioritization of resources. The organizational repository stores personal information of all members of the company along with their positions, roles, and units within the organization, information about skills, salary or hiring date, and all the data that is not related to participation in $\mathrm{BP}$ activities. The worklist repository stores the worklists of all resources in the organization. Finally, the history repository stores the event log of past process executions. The organizational repository is manually updated, whereas the worklist repository and the history repository are updated by the BPMS, which in our implementation is Activiti. The eval functions can be divided into the following four categories according to the repository they use to evaluate the domain concept:

Quantitative organizational evaluations: They are used to evaluate concepts that represent quantitative properties stored in the organizational repository such as personal (e.g., age) or professional information (e.g., salary).

Qualitative organizational evaluations: They are used to evaluate concepts that represent qualitative properties stored in the organizational repository including organizational information and skills. The implementation of this type of evaluations leverages CRISTAL [5] to resolve RAL expressions and obtain the set of resources.

Worklist evaluations: They are used to evaluate concepts that represent quantitative properties related to the size of the worklist. They interact with the BPMS to obtain the information about them.

History evaluations: They are used to evaluate concepts that represent quantitative properties about the participation of resources in past process activity executions. This evaluation also accesses the history of the BP.

\section{Related Work}

We next present a summary of the pros and cons of the current approaches for preference modeling in different domains, followed by an analysis of current support for resource prioritization in Business Process Management (BPM)

Preference Modeling. There are several formalisms that can be used to represent preferences in different fields [15]. Quantitative preferences modeled as utility or scoring functions have been widely used in economics and operations 
research [16, 17], as well as in web systems [18, 19]. This approach solves the multiple criteria decision making by transforming it to aggregated scoring functions. However, these functions are difficult to define by users, and not all the preferences that are strict partial orders can be represented [20]. In artificial intelligence research, solutions have been focused on defining preferences in a qualitative way, easier to understand and more natural to define by humans [21]. These preference models offer facilities to define preferences as a set of statements or terms that are contextually related. In database research, there are also several solutions, for instance, using top-k or skyline algorithms to obtain the best search items according to a stated preference [22, 20]. These preference models usually offer qualitative facilities to define preferences, though their implementation usually leads to large result sets that do not discriminate well between items to be ordered [15]. We have chosen SOUP [4] because it is a hybrid approach, as it combines quantitative and qualitative facilities to define preferences. Furthermore, it is independent of the domain, so it is suitable for resource prioritization. Indeed, the expressiveness of the model is semantically close to BP modeling, which enables its interoperability with other resource allocation solutions.

Resource Prioritization in Business Processes. Regarding the prioritization of resources in BPM] we have studied the support provided by the specifications BPMN 2.0, BPEL4People and WS-HumanTask; the BPMS Activiti and YAWL [10]; and the product suites WebSphere MQ and ARIS systems, concerning preference definition and resource ranking. We find that they neither provide support for preference specification nor for ranking of potential performers in order to prioritize resource allocation. Crowdsourcing systems, which outsource the execution of activities to the crowd, usually rely on a fixed set of features such as skills, location, certification, cost or reputation to implement priotization [23], or they use some auction or competition mechanism to select the best worker 24]. However, the prioritization mechanism is defined in the system for all the activities and cannot be customized according to other criteria.

The importance of ranking resources is also emphasized in other recent work. In [25], the authors define a resource visualization concept that is aimed to support resource allocation using three different metrics to recommend work distribution. The distribution of work is addressed in [26] trying to keep the balance between quality and performance. In [27], six resource allocation mechanisms are compared with regard to suitability, urgency, conformance and availability. Although all of them agree on the need of dynamic work allocation to adapt to the evoluting needs of organizations, they do not deal with preference modeling itself. Some other solutions approach this problem from a process mining perspective, focusing on providing recommendation from information inferred from event logs [28 31]. A resource manager is finally responsible for making the final decisions for allocation. Altogether, the work presented in this paper generalizes and complements the current support in the field of BPM regarding preference modeling. It would be interesting to combine it, e.g., with visualization support as proposed in [25, 31]. 


\section{Conclusions and Future Work}

In this paper, we addressed the problem of integrating priorities into resource assignment and allocation. To this end, we extended concepts from preference modeling and combined them with resource management techniques.

The main advantage of our solution is that it provides a mechanism to define a wide variety of different types of preferences while being independent of both the properties used in the preferences and the knowledge base that contains the information about them. These features make it easier to accommodate the different requirements several organizations may have. This is a significant difference with respect to the support provided by current systems, which are defined to deal with a specific set of properties. Furthermore, the expressiveness of the preferences that can be defined with our approach outperforms the current support in the BPM field regarding priority-based allocation.

We plan to extend the preference model to support complex cases involving the agenda of the resources in order to allow expressing and using preferences referring to the expected end time of an activity. We also want to explore how a similar technique could be applied to the distribution of work to resources, i.e., in the opposite direction. Activity priority might be considered in that case.

\section{References}

1. Russell, N., van der Aalst, W.M.P., ter Hofstede, A.H.M., Edmond, D.: Workflow Resource Patterns: Identification, Representation and Tool Support. In: Pastor, Ó., Falcão e Cunha, J. (eds.) CAiSE 2005. LNCS, vol. 3520, pp. 216-232. Springer, Heidelberg (2005)

2. van der Aalst, W.M.P., ter Hofstede, A.H.M.: YAWL: Yet Another Workflow Language. Inf. Syst. 30(4), 245-275 (2005)

3. Tan, H., van der Aalst, W.M.P.: Implementation of a YAWL Work-List Handler based on the Resource Patterns. In: CSCWD 2006, pp. 1-6 (2006)

4. García, J.M., Ruiz, D., Ruiz-Cortés, A.: A Model of User Preferences for Semantic Services Discovery and Ranking. In: Aroyo, L., Antoniou, G., Hyvönen, E., ten Teije, A., Stuckenschmidt, H., Cabral, L., Tudorache, T. (eds.) ESWC 2010, Part II. LNCS, vol. 6089, pp. 1-14. Springer, Heidelberg (2010)

5. Cabanillas, C., del Río-Ortega, A., Resinas, M., Ruiz-Cortés, A.: CRISTAL: Collection of Resource-centrIc Supporting Tools And Languages. In: BPM 2012 Demos, vol. 940, pp. 51-56 (2012)

6. García, J.M., Junghans, M., Ruiz, D., Agarwal, S., Ruiz-Cortés, A.: Integrating Semantic Web Services Ranking Mechanisms Using a Common Preference Model. Knowledge-Based Systems 49, 22-36 (2013)

7. Cabanillas, C., Resinas, M., Ruiz-Cortés, A.: Defining and Analysing Resource Assignments in Business Processes with RAL. In: Kappel, G., Maamar, Z., MotahariNezhad, H.R. (eds.) ICSOC 2011. LNCS, vol. 7084, pp. 477-486. Springer, Heidelberg (2011)

8. Strembeck, M., Mendling, J.: Modeling process-related RBAC models with extended UML activity models. Inf. Softw. Technol. 53, 456-483 (2011)

9. Awad, A., Grosskopf, A., Meyer, A., Weske, M.: Enabling Resource Assignment Constraints in BPMN. tech. rep., BPT (2009)

10. Adams, M.: The Resource Service. In: Modern Business Process Automation, pp. 261-290 (2010) 
11. Davey, B.A., Priestley, H.A.: Introduction to Lattices and Order, 2nd edn. Cambridge University Press (2002)

12. Cabanillas, C., Resinas, M., Ruiz-Cortés, A.: RAL: A high-level user-oriented resource assignment language for business processes. In: Daniel, F., Barkaoui, K., Dustdar, S. (eds.) BPM Workshops 2011, Part I. LNBIP, vol. 99, pp. 50-61. Springer, Heidelberg (2012)

13. Russell, N., ter Hofstede, A., Edmond, D., van der Aalst, W.M.P.: Workflow Resource Patterns. tech. rep., BETA, WP 127, Eindhoven University of Technology (2004)

14. Cormen, T.H., Leiserson, C.E., Rivest, R.L., Stein, C.: Introduction to algorithms. MIT press (2001)

15. Domshlak, C., Hüllermeier, E., Kaci, S., Prade, H.: Preferences in ai: An overview. Artif. Intell. 175(7-8), 1037-1052 (2011)

16. Fishburn, P.C.: Utility theory for decision making. Wiley (1970)

17. Keeney, R.L., Raiffa, H.: Decisions with multiple objectives: Preferences and value tradeoffs. Cambridge Univ Press (1993)

18. Agrawal, R., Wimmers, E.L.: A Framework for Expressing and Combining Preferences. In: SIGMOD Conference, pp. 297-306 (2000)

19. Zeng, L., Benatallah, B., Ngu, A.H.H., Dumas, M., Kalagnanam, J., Chang, H.: QoS-Aware Middleware for Web Services Composition. IEEE Trans. Software Eng. 30(5), 311-327 (2004)

20. Chomicki, J.: Preference formulas in relational queries. ACM Trans. Database Syst. 28(4), 427-466 (2003)

21. Boutilier, C., Brafman, R.I., Domshlak, C., Hoos, H.H., Poole, D.: CP-nets: A Tool for Representing and Reasoning with Conditional Ceteris Paribus Preference Statements. J. Artif. Intell. Res (JAIR) 21, 135-191 (2004)

22. Kießling, W.: Foundations of Preferences in Database Systems. In: VLDB, pp. 311-322 (2002)

23. Vukovic, M.: Crowdsourcing for Enterprises. In: SERVICES, pp. 686-692 (2009)

24. Satzger, B., Psaier, H., Schall, D., Dustdar, S.: Auction-based crowdsourcing supporting skill management. Inf. Syst. 38(4), 547-560 (2013)

25. De Leoni, M., Adams, M., van der Aalst, W.M.P., Ter Hofstede, A.H.M.: Visual support for work assignment in process-aware information systems: Framework formalisation and implementation. Decis. Support Syst. 54(1), 345-361 (2012)

26. Kumar, A., van der Aalst, W.M.P., Verbeek, E.M.W.: Dynamic Work Distribution in Workflow Management Systems: How to Balance Quality and Performance. J. Manage. Inf. Syst. 18(3), 157-193 (2002)

27. Reijers, H.A., Jansen-Vullers, M.H., zur Muehlen, M., Appl, W.: Workflow management systems + swarm intelligence $=$ dynamic task assignment for emergency management applications. In: Alonso, G., Dadam, P., Rosemann, M. (eds.) BPM 2007. LNCS, vol. 4714, pp. 125-140. Springer, Heidelberg (2007)

28. Liu, T., Cheng, Y., Ni, Z.: Mining event logs to support workflow resource allocation. Know.-Based Syst. 35, 320-331 (2012)

29. Liu, Y., Wang, J., Yang, Y., Sun, J.: A semi-automatic approach for workflow staff assignment. Comput. Ind. 59(5), 463-476 (2008)

30. Rinderle-Ma, S., van der Aalst, W.M.P.: Life-Cycle Support for Staff Assignment Rules in Process-Aware Information Systems. Department of Technology Management, Eindhoven University of Technology (2007)

31. Bose, R.P.J.C., van der Aalst, W.M.P.: Process Mining Applied to the BPI Challenge 2012: Divide and Conquer While Discerning Resources. In: La Rosa, M., Soffer, P. (eds.) BPM Workshops 2012. LNBIP, vol. 132, pp. 221-222. Springer, Heidelberg (2013) 